\title{
On a unified approach to the law of the iterated logarithm for martingales
}

\section{P.G. Hall and C.C. Heyde}

\begin{abstract}
There are two distinct approaches in the literature to framing a version of the law of the iterated logarithm for martingales. One involves norming by constants, using the martingale variance and the other involves norming by random variables, using the sums of conditional variances of the increments, given their past. In this paper a portmanteau approach is provided, still based on the Skorokhod representation of the martingale, but involving normalization by more general random variables. This extends the functional forms of all the previously existing results.
\end{abstract}

\section{Introduction and results}

The literature contains various laws of the iterated logarithm for martingales which are not directly comparable. There are versions based on norming by constants (for example, Heyde and Scott [1]) and versions based on norming by random variables (for example, Strassen [7], Stout [5]). The former use variances and the latter, conditional variances. Here we employ a Skorokhod representation approach, based on the law of the iterated logarithm for brownian motion, to relate the earlier results.

Let $\phi$ be the real-valued function on $(e, \infty)$ defined by

$$
\phi(t)=(2 t \log \log t)^{\frac{3}{2}} .
$$

If $W(t)(t \geq 0)$ is standara brownian motion then (Lévy [3])

Received 1 March 1976. 
(1)

$$
\underset{t \rightarrow \infty}{\lim \sup } W(t) / \phi(t)=+1 \text { almost surely, }
$$

$$
\underset{t \rightarrow \infty}{\lim \inf } W(t) / \phi(t)=-1 \text { almost surely. }
$$

If $\left\{t_{n}, n \geq 1\right\}$ is a sequence of constants increasing to $\infty$ then (1) implies that

$$
\begin{aligned}
& \underset{n \rightarrow \infty}{\lim \sup } W\left(t_{n}\right) / \phi\left(t_{n}\right) \leq+1 \text { almost surely, } \\
& \underset{n \rightarrow \infty}{\lim \inf } W\left(t_{n}\right) / \phi\left(t_{n}\right) \geq-1 \text { almost surely. }
\end{aligned}
$$

If the points of the sequence $\left\{t_{n}\right\}$ are close enough together then equality holds throughout (2) with probability 1 . In fact, if $\left\{T_{n}, n \geq 1\right\}$ is any sequence of random variables satisfying

$$
T_{n}>e, T_{n}+\infty \text { almost surely and } T_{n+1}^{-1} T_{n} \stackrel{\text { a.s. }}{\longrightarrow} 1
$$

then (1) implies that

$$
\begin{aligned}
& \underset{n \rightarrow \infty}{\lim \sup } W\left(T_{n}\right) / \phi\left(T_{n}\right)=1 \quad \text { almost surely } \\
& \underset{n \rightarrow \infty}{\lim \inf } W\left(T_{n}\right) / \phi\left(T_{n}\right)=-1 \text { almost surely, }
\end{aligned}
$$

and so the variables $S_{n}=W\left(T_{n}\right)$ satisfy the law of the iterated logarithm with a random norming.

Now let $\left\{s_{n}, F_{n}, n \geq 1\right\}$ be a zero-mean, square-integrable martingale on the probability space $(\Omega, F, P)$, where $F_{0}=\{\phi, \Omega\}$ and $F_{n}$ is the o-field generated by $S_{j}, 0 \leq j \leq n$. Let $S_{0}=X_{0}=0$ and $S_{n}=\sum_{1}^{n} X_{k}$. We shall wite

$$
v_{n}^{2}=\sum_{1}^{n} E\left(x_{k}^{2} \mid F_{k-1}\right), \quad v_{n}^{2}=\sum_{1}^{n} x_{k}^{2}, \quad \varepsilon_{n}^{2}=E S_{n}^{2}=E V_{n}^{2}
$$

and $\left\{w_{n}, n \geq I\right\}$ will denote a non-decreasing sequence of positive random variables such that $w_{1}^{2}>e$. By extending the original probability space 
if necessary, we may suppose that there exists a brownian motion $W$ and a non-decreasing sequence of non-negative variables $\left\{T_{n}, n \geq 1\right\}$ defined on our probability space such that $S_{n}=W\left(T_{n}\right)$ almost surely for all $n$ (see the statement of the skorokhod representation theorem in Strassen [7], Theorem 4.3). If the $T_{n}$ satisfy condition (3) then (4) immediately gives us a law of the iterated logarithm for $\left\{s_{n}, n \geq 0\right\}$.

Unfortunately the $T_{n}$ are quite difficult to compute from the martingale $\left\{S_{n}, F_{n}\right\}$, and the law is not very meaningful unless we can replace $T_{n}$ by a variable $W_{n}^{2}$ which can be expressed as a function of the differences $x_{1}, x_{2}, \ldots ; w_{n}^{2}$ should satisfy

$$
T_{n}^{-1} w_{n}^{2} \stackrel{\text { a.s. }}{\longrightarrow} 1
$$

Strassen [6] formulated a functional law of the iterated logarithm which extends the law in (4). Consider the metric space $(C, \rho)$ of all real-valued continuous functions on $[0,1]$ with

$$
\rho(x, y)=\sup _{0 \leq t \leq 1}|x(t)-y(t)| \text { for } x, y \in C \text {. }
$$

Let $K$ be the set of absolutely continuous $x \in C$ such that $x(0)=0$ and

$$
\int_{0}^{1} \dot{x}(t)^{2} d t \leq 1
$$

where $\dot{x}$ denotes the derivative of $x$ determined almost everywhere with respect to Lebesgue measure. For $u \in[0,1]$ define

$$
\tau=z(n, u)=\max \left\{j \leq n \mid w_{j}^{2} \leq u w_{n}^{2}\right\} \quad(=0 \text { if the set is empty })
$$

and

$$
\mu_{n}(u)=\left[\phi\left(w_{n}^{2}\right)\right]^{-1}\left[S_{\eta}+\left(u w_{n}^{2}-w_{\eta}^{2}\right)\left(w_{l+1}^{2}-w_{\eta}^{2}\right)^{-1} x_{\eta+1}\right],
$$

where $S_{n}=W\left(T_{n}\right)$ and $X_{n}=S_{n}-S_{n-1} \quad\left(s_{0}=0\right)$. 
THEOREM A. If (3) and (5) hold then $\left\{\mu_{n}, n \geq 1\right\}$ is relatively compact in $c[0,1]$ and the set of $i$ ts almost sure limit points coincides with $K$.

(Here we do not assume that $\left\{S_{n}, F_{n}, n \geq 0\right\}$ is a martingale, although we do in all the work which follows.)

If we are to use Theorem A to establish a law of the iterated logarithm for martingales, we must find conditions under which $T_{n}$ can be approximated by functions $w_{n}^{2}$ of the martingale differences. It is convenient to work with martingales whose differences are truncated, and hence our technique is to approximate to $\left\{s_{n}\right\}$ by a truncated martingale $\left\{S_{n}^{*}\right\}=\left\{w\left(T_{n}^{*}\right)\right\}$, and then find functions $w_{n}^{2}$ which are close to $T_{n}^{*}$. our main result is given in the following theorem.

THEOREM 1. Let $\left\{z_{n}, n \geq 1\right\}$ be a sequence of non-negative random variables and suppose that $z_{n}$ and $W_{n}$ are $F_{n-1}$-measurable. If

(6) $\lim _{n \rightarrow \infty}\left[\phi\left(w_{n}^{2}\right]\right]^{-1} \sum_{I}^{n}\left\{x_{j} I\left(\left|x_{j}\right|>z_{j}\right)-E\left(x_{j} I\left(\left|x_{j}\right|>z_{j}\right) \mid F_{j-1}\right)\right\}=0$ almost surely,

(7) $\lim _{n \rightarrow \infty} W_{n}^{-2} \sum_{1}^{n}\left[E\left(x_{j}^{2} I\left(\left|x_{j}\right| \leq z_{j}\right) \mid F_{j-1}\right)-\left\{E\left(x_{j} I\left(\left|x_{j}\right| \leq z_{j}\right) \mid F_{j-1}\right)\right\}^{2}\right]=1$ almost surely,

$$
\sum_{1}^{\infty} w_{j}^{-4} E\left(X_{j}^{4} I\left(\left|x_{j}\right| \leq z_{j}\right) \mid F_{j-1}\right)<\infty \text { almost surely, }
$$

and

$$
\lim _{n \rightarrow \infty} W_{n+1}^{-1} W_{n}=1 \text { almost surely and } W_{n} \rightarrow \infty \text { almost surely, }
$$

then the conclusions of Theorem A hold.

Two obvious candidates for $W_{n}$ for use in this theorem are $v_{n} \vee 2$ and $U_{n-1} \vee 2$. These lead to equivalent results if $\lim _{n \rightarrow \infty} V_{n}^{-2} U_{n}^{2}=1$ almost 
surely, which would commonly be the case; but interestingly enough this would not universally be true. When $V_{n}$ is used in the norming the condition (7) simplifies usefully, giving the following result.

COROLLARY 1. Let $\left\{z_{n}, n \geq 1\right\}$ be as in Theorem 1. The conclusions of Theorem A hold for $w_{n}=V_{n} \vee 2$ if

(10) $\lim _{n \rightarrow \infty}\left[\phi\left(v_{n}^{2}\right)\right]^{-1} \sum_{1}^{n}\left\{x_{j} I\left(\left|x_{j}\right|>z_{j}\right)-\right.$

$$
\left.-E\left(X_{j} I\left(\left|X_{j}\right|>z_{j}\right) \mid F_{j-1}\right)\right\}=0 \text { almost surely, }
$$

$$
\begin{gathered}
\lim _{n \rightarrow \infty} V_{n}^{-2} \sum_{1}^{n} E\left(x_{j}^{2} I\left(\left|x_{j}\right|>z_{j}\right) \mid F_{j-1}\right)=0 \text { almost sure } y_{y}, \\
\quad \sum_{1}^{\infty} v_{j}^{-4} E\left(x_{j}^{4} I\left(\left|x_{j}\right| \leq z_{j}\right) \mid F_{j-1}\right)<\infty \text { almost sure } z_{y},
\end{gathered}
$$

and

$$
\lim _{n \rightarrow \infty} V_{n+1}^{-1} V_{n}=1 \text { almost surely, } V_{n} \rightarrow \infty \text { almost surely. }
$$

The next corollary extends the main result (Theorem 1) of Heyde and Scott [1] which deals with the case $\eta^{2}=1$ almost surely. It highlights the restrictiveness of a constant norming form.

COROLLARY 2. If for some almost surely finite and non-zero random variable $\mathrm{n}^{2}$ we have

$$
\begin{gathered}
s_{n}^{-2} U_{n}^{2} \stackrel{\text { a.s. }}{\longrightarrow} \eta^{2} \text { and } s_{n}+\infty, \\
\sum_{i}^{\infty} s_{j}^{-1} E\left[\left|x_{j}\right| I\left(\left|x_{j}\right|>\varepsilon s_{j}\right)\right]<\infty \text {, for all } \varepsilon>0,
\end{gathered}
$$

and

$$
\sum_{I}^{\infty} s_{j}^{-4} E\left[X_{j}^{4} I\left(\left|X_{j}\right| \leq \delta s_{j}\right)\right]<\infty \text {, for some } \delta>0 \text {, }
$$

then the conclusions of Theorem A hold for $W_{n}=U_{n-1} \vee 2$. 
The final corollary presents a portmanteau form for the sufficient conditions.

COROLLARY 3. Let $f$ and $g$ be positive non-decreasing functions on $(0, \infty)$ such that $t^{-1} f(t) \downarrow 0, t^{-1} g(t) \downarrow 0$ as $t$ increases and

$$
\int_{1}^{\infty} t^{-2} f(t) d t<\infty
$$

If $V_{n} \rightarrow \infty$ almost surely as $n \rightarrow \infty$ and

$$
\sum_{1}^{\infty}\left[g\left(v_{j}^{2}\right)\right]^{-1} E\left(x_{j}^{2} I\left(x_{j}^{2}>f\left(v_{j}^{2}\right)\right) \mid F_{j-I}\right)<\infty \text { almost surely, }
$$

then the conclusions of Theorem A hold for $w_{n}=V_{n} \vee 2$.

This result can be compared with Corollary 4.5 of Strassen [7] which deals with the case $f(t)=g(t)=t(\log t)^{-5}, t>e$, and with Theorem 5.1 of Jain, Jogdeo and Stout [2] which deals with the case

$f(t)=t(\log t)^{-1}(\log \log t)^{-6}, g(t)=t(\log \log t)^{-2}, t>e$. These results, however, are concerned with the integral test for upper and lower functions which, even in the case of independent variables, requires the imposition of slightly more stringent conditions than for the classical law of the iterated logarithm itself. Theorem 3 of Stout [5] essentially deals with the case $f(t)=g(t)=o\left(t(\log \log t)^{-1}\right), t>e$, but obtains the classical and not the functional law of the iterated logarithm.

\section{Proofs}

Proof of Theorem A. Define $\mu(u)$ on $[0, \infty)$ by

$$
\mu(u)=s_{p}+\left(u-w_{p}^{2}\right)\left(w_{p+1}^{2}-w_{p}^{2}\right)^{-1} x_{p+1},
$$

where

$$
p=p(u)=\max \left\{j \mid w_{j}^{2} \leq u\right\}
$$

Then 


$$
\mu_{n}(u)=\left[\phi\left(w_{n}^{2}\right]\right]^{-1} \mu\left(w_{n}^{2} u\right),
$$

and so in view of Corollary 1 of Strassen [6], it suffices to prove that

$$
\lim _{t \rightarrow \infty}[\phi(t)]^{-1} \sup _{u \leq t}|\mu(u)-W(u)|=0 \text { almost surely. }
$$

Now, (3) and (5) imply that $W_{n+1}^{-2} W_{n}^{2} \stackrel{\text { a.s. }}{\longrightarrow} 1$ and hence

$$
1 \geq u^{-1} w_{p(u)}^{2} \geq w_{p(u)+1}^{-2} w_{p(u)}^{2} \stackrel{\text { a.s. }}{\longrightarrow} 1 \text { as } u \rightarrow \infty \text {, }
$$

so that

$$
u^{-1} W_{p(u)}^{2} \stackrel{\text { a.s. }}{\longrightarrow} I \text { as } u \rightarrow \infty
$$

Similarly,

$$
u^{-1} W_{p(u)+1}^{2} \stackrel{\text { a.s. }}{\longrightarrow} 1 \text { as } u \rightarrow \infty \text {. }
$$

Combining these and (5),

$$
u^{-1} T_{p(u) \stackrel{\text { a.s. }}{\longrightarrow} 1} \text { and } u^{-I_{T}} p(u)+1 \stackrel{\text { a.s. }}{\longrightarrow} 1 \text { as } u \rightarrow \infty \text {. }
$$

Since

$$
|\mu(u)-W(u)| \leq \max \left\{\left|W\left(T_{p(u)}\right)-W(u)\right|,\left|W\left(T_{p(u)+1}\right)-W(u)\right|\right\},
$$

then result (19) follows as in Strassen's proof on page 217 of [6].

Proof of Theorem 1. The proof is based on that of Strassen [6] and follows that of Heyde and Scott [1].

Define

(20) $\tilde{X}_{j}=X_{j} I\left(c_{j}<\left|X_{j}\right| \leq z_{j}\right)+\frac{1}{2} X_{j} I\left(\left|X_{j}\right| \leq c_{j}\right)$

$$
+\frac{z}{2} \operatorname{sgn}\left(x_{j}\right) c_{j}\left(1+z_{j}\left|x_{j}\right|^{-1}\right) I\left(\left|x_{j}\right|>z_{j}\right)
$$

where $\left\{c_{j}, j \geq 1\right\}$ is a monotone sequence of positive constants with $c_{j} \rightarrow 0$ as $j \rightarrow \infty$ so fast that

$$
\sum_{1}^{\infty} c_{j}<\infty, \sum_{l}^{\infty} c_{j} Z_{j} W_{j}^{-2}<\infty \text { almost surely. }
$$


(If $z_{j}(\omega)<c_{j}$, let $\left.I\left(c_{j}<\left|X_{j}\right| \leq z_{j}\right)(\omega)=0.\right)$ set also

$$
X_{j}^{*}=\tilde{X}_{j}-E\left(\tilde{X}_{j} \mid F_{j-1}\right) \text {. }
$$

It is easily checked that $\tilde{X}_{1}, \ldots, \tilde{X}_{n}$ and hence $X_{1}^{*}, \ldots, X_{n}^{*}$ also genergte the o-field $F_{n}$.

$$
\begin{gathered}
\text { Write } S_{n}^{*}=\sum_{1}^{n} x_{j}^{*}, \quad V_{n}^{*^{2}}=\sum_{1}^{n} E\left(x_{j}^{*} \mid F_{j-1}\right), \text { and if } u \in[0,1], \text { let } \\
\mu_{n}^{*}(u)=\left[\phi\left(w_{n}^{2}\right]^{-1}\left[S_{l}^{*}\left(u w_{n}^{2}-w_{l}^{2}\right)\left(w_{l+1}^{2}-w_{l}^{2}\right)^{-1} x_{l+1}^{*}\right] .\right.
\end{gathered}
$$

We use (20) to obtain

$$
\left|X_{j}-X_{j}^{*}\left\{X_{j} I\left(\left|X_{j}\right|>z_{j}\right)-E\left(X_{j} I\left(\left|X_{j}\right|>z_{j}\right) \mid F_{j-1}\right)\right\}\right| \leq 2 c_{j},
$$

and then

$$
\begin{aligned}
\sup _{0 \leq u \leq 1}\left|\mu_{n}(u)-\mu_{n}^{*}(u)\right| \leq\left[\phi\left(w_{n}^{2}\right)\right]^{-1} \sup _{1 \leq r \leq n}\left|\sum_{j=1}^{p}\left(x_{j}-X_{j}^{*}\right)\right| \\
\leq\left[\phi\left(w_{n}^{2}\right]^{-1} \sup _{1 \leq r^{\prime} \leq n}\left|\sum_{1}^{p}\left\{x_{j} I\left(\left|x_{j}\right|>z_{j}\right)-E\left(x_{j} I\left(\left|x_{j}\right|>z_{j}\right) \mid F_{j-1}\right)\right\}\right|+\right. \\
+\left[\phi\left(w_{n}^{2}\right)\right]^{-1} \sum_{1}^{n} 2 c_{j}
\end{aligned}
$$

$\stackrel{\text { a.s. }}{\longrightarrow} 0$

as $n \rightarrow \infty$, in view of (6).

Next we introduce the Skorokhod representation (see Strassen [7], Theorem 4.3). By extending the original probability space if necessary, we may suppose that there exists a brownian motion $W$ and a sequence $\left\{T_{n}^{*}, n \geq 1\right\}$ of non-negative random variables defined on our probability space such that $S_{n}^{*}=W\left(T_{n}^{*}\right)$ almost surely for all $n$. Let $t_{n}=T_{n}^{*}-T_{n-1}^{*}, n \geq 1 \quad\left(T_{0}=0\right)$. If $G_{n}$ is the $\sigma$-field generated by $X_{1}, \ldots, X_{n}$ and $W(u)$ for $u<T_{n}^{*}$, then $t_{n}$ is $G_{n}$-measurable,

$$
E\left(t_{n} \mid G_{n-1}\right)=E\left(X_{n}^{\star^{2}} \mid G_{n-1}\right)=E\left(X_{n}^{\star^{2}} \mid F_{n-1}\right) \text { almost surely, }
$$

and for some constant $L$, 


$$
E\left(t_{n}^{2} \mid G_{n-1}\right) \leq L E\left(X_{n}^{*^{4}} \mid G_{n-1}\right)=L E\left(X_{n}^{*^{4}} \mid F_{n-1}\right) \text { almost surely. }
$$

In view of (9), (21) and Theorem A, it suffices to prove that

$$
W_{n}^{-2} T_{n}^{*} \stackrel{\text { a.s. }}{\longrightarrow} 1
$$

To this end we first show that

$$
T_{n}^{*}-V_{n}^{*^{2}}=o\left(W_{n}^{2}\right) \text { almost surely as } n \rightarrow \infty
$$

Since

$$
\begin{aligned}
E\left(X_{j}^{*} \mid F_{j-1}\right) & =E\left(\tilde{X}_{j}^{4} \mid F_{j-1}\right)-4 E\left(\tilde{X}_{j}^{3} \mid F_{j-1}\right) E\left(\tilde{X}_{j} \mid F_{j-1}\right) \\
& +6 E\left(\tilde{X}_{j}^{2} \mid F_{j-1}\right)\left[E\left(\tilde{x}_{j} \mid F_{j-1}\right)\right]^{2}-3\left[E\left(\tilde{x}_{j} \mid F_{j-1}\right)\right]^{4} \\
& \leq 11 E\left(\tilde{X}_{j}^{4} \mid F_{j-1}\right) \\
& \leq 11 E\left(x_{j}^{4} I\left(\left|x_{j}\right| \leq z_{j}\right) \mid F_{j-1}\right)+11 c_{j}^{4},
\end{aligned}
$$

we have from (8) that

$$
\sum_{1}^{\infty} W_{j}^{-4} E\left(X_{j}^{*^{4}} \mid F_{j-1}\right)<\infty \text { almost surely }
$$

and hence, using Proposition IV.6.2, page 148 of Neveu [4],

$$
\sum_{1}^{n}\left[t_{j}-E\left(t_{j} \mid G_{j-1}\right)\right]=o\left(w_{n}^{2}\right) \text { almost surely, }
$$

which is equivalent to (23).

Next, we have

$$
\begin{aligned}
& \left|E\left(\tilde{X}_{j}^{2} \mid F_{j-1}\right)-E\left(X_{j}^{2} I\left(\left|x_{j}\right| \leq z_{j}\right) \mid F_{j-1}\right)\right| \leq c_{j}^{2}, \\
& E\left(X_{j}^{x^{2}} \mid F_{j-1}\right)=E\left(\tilde{X}_{j}^{x^{2}} \mid F_{j-1}\right)-\left(E\left(\tilde{X}_{j} \mid F_{j-1}\right)\right)^{2},
\end{aligned}
$$

and

$$
\left|E\left(\tilde{X}_{j} \mid F_{j-1}\right)-E\left(X_{j} I\left(\left|X_{j}\right| \leq z_{j}\right) \mid F_{j-1}\right)\right| \leq c_{j} .
$$

Then, using (26), 
(27) $\sum_{1}^{n}\left[\left\{E\left(\tilde{X}_{j} \mid F_{j-1}\right)\right\}^{2}-\left\{E\left(x_{j} I\left(\left|x_{j}\right| \leq z_{j}\right) \mid F_{j-1}\right)\right\}^{2}\right]=$ $=o\left(w_{n}^{2}\right)$ almost surely

since

$$
\sum_{1}^{n} c_{j}\left|E\left(x_{j} I\left(\left|x_{j}\right| \leq z_{j}\right) \mid F_{j-1}\right)\right|=o\left(w_{n}^{2}\right) \text { almost surely, }
$$

by virtue of $\sum_{1}^{\infty} c_{j}{ }^{2} W_{j}^{-2}<\infty$ almost surely and Kronecker's Lemma. Hence, from (7) in conjunction with (24), (25), and (27), we have

$$
v_{n}^{t^{2}}-w_{n}^{2}=o\left(w_{n}^{2}\right) \text { almost surely. }
$$

Combined with (23), this establishes (22) and completes the proof of Theorem 1 .

Proof of Corollary 1. Take $W_{n}=V_{n} \vee 2$ for all $n$. Conditions (6), (8), and (9) translate immediately into (10), (12), and (13). To check condition (7) it suffices to show that

$$
\lim _{n \rightarrow \infty} V_{n}^{-2} \sum_{1}^{n}\left\{E\left(x_{j} I\left(\left|x_{j}\right| \leq z_{j}\right) \mid F_{j-1}\right)\right\}^{2}=0 \text { almost surely. }
$$

That (28) is indeed satisfied follows from

$$
\begin{aligned}
& E\left(X_{j} I\left(\left|X_{j}\right| \leq z_{j}\right) \mid F_{j-1}\right)=-E\left(X_{j} I\left(\left|x_{j}\right|>z_{j}\right) \mid F_{j-1}\right) \text { almost surely, } \\
& \left\{E\left(X_{j} I\left(\left|X_{j}\right|>z_{j}\right) \mid F_{j-1}\right)\right\}^{2} \leq E\left(X_{j}^{2} I\left(\left|X_{j}\right|>z_{j}\right) \mid F_{j-I}\right) \text { almost surely, }
\end{aligned}
$$

and (11).

Proof of Corollary 2. We use Theorem 1 and take $z_{j}=\delta s_{j}$, $w_{j}=U_{j-1} v_{2}, j>1$.

The condition (15), together with Kronecker's Lemma, gives 
(29)

$$
s_{n}^{-1} \sum_{1}^{n}\left|X_{j}\right| I\left(\left|x_{j}\right|>\varepsilon s_{j}\right) \stackrel{\text { a.s. }}{\longrightarrow} 0
$$

$$
s_{n}^{-1} \sum_{1}^{n} E\left(\left|x_{j}\right| I\left(\left|x_{j}\right|>\varepsilon s_{j}\right) \mid F_{j-1}\right) \stackrel{\text { a.s. }}{\longrightarrow} 0 \text {, }
$$

as $n \rightarrow \infty$ for any $\varepsilon>0$, so that in particular, for any $\varepsilon>0$,

$$
s_{n}^{-I} \sup _{j \leq n}\left|X_{j}\right| \leq \varepsilon+s_{n}^{-I} \sum_{1}^{n}\left|X_{j}\right| I\left(\left|X_{j}\right|>\varepsilon s_{j}\right) \stackrel{\text { a.s. }}{\longrightarrow} \varepsilon .
$$

Hence

$$
s_{n}^{-2} \sup _{j \leq n} x_{j}^{2} \stackrel{\text { a.s. }}{\longrightarrow} 0
$$

and in view of (14),

$$
U_{n}^{-2} \sup _{j \leq n} X_{j}^{2} \stackrel{\text { a.s. }}{\longrightarrow} 0
$$

which implies (9). We can now observe from (29) that (6) holds, while (16) ensures (8).

Finally, (16) yields via an application of Proposition IV.6.1 of [4],

$$
\text { (31) } \begin{aligned}
\lim _{n \rightarrow \infty} s_{n}^{-2} \sum_{1}^{n}\left[x_{j}^{2} I\left(\left|x_{j}\right| \leq \delta s_{j}\right)-E\left(x_{j}^{2} I\left(\left|x_{j}\right| \leq \delta s_{j}\right) \mid F_{j-1}\right]\right. & = \\
& =0 \text { almost surely, }
\end{aligned}
$$

while

(32) $s_{n}^{-2} \sum_{1}^{n} x_{j}^{2} I\left(\left|x_{j}\right|>\delta_{s_{j}}\right) \leq$

$$
\leq\left(\varepsilon_{n}^{-2} \sup _{j \leq n}\left|X_{j}\right|\right) s_{n}^{-1} \sum_{I}^{n}\left|X_{j}\right| I\left(\left|x_{j}\right|>\delta_{j}\right) \stackrel{\text { a.s. }}{\longrightarrow} 0
$$

using (29) and (30). We deduce from (31) and (32) that

$$
\lim _{n \rightarrow \infty} s_{n}^{-2} \sum_{I}^{n} E\left(X_{j}^{2} I\left(\left|x_{j}\right| \leq \delta s_{j}\right) \mid F_{j-1}\right)=n^{2} \text { almost surely, }
$$

and condition (7) follows in view of (29). This completes the proof. 
Proof of Corollary 3 . We take $z_{j}^{2}=f\left(v_{j}^{2}\right)$ and check the conditions of Corollary 1 .

A sufficient condition for (10) is, using Proposition IV.6.2 of [4],

$$
\sum_{1}^{\infty}\left[\phi\left(v_{j}^{2}\right]\right]^{-2} E\left(X_{j}^{2} I\left(x_{j}^{2}>f\left(V_{j}^{2}\right)\right) \mid F_{j-1}\right)<\infty \text { almost surely, }
$$

which holds in view of (18) since $t^{-1} g(t) \rightarrow 0$ as $t \rightarrow \infty$. Furthermore, that (11) holds follows immediately from (18) and an application of Kronecker's Lemma.

To check (12), we have

$$
\begin{aligned}
\sum_{2}^{\infty} v_{j}^{-4} E\left(X_{j}^{4} I\left(x_{j}^{2} \leq f\left(v_{j}^{2}\right)\right) \mid F_{j-1}\right) & \leq \sum_{2}^{\infty} v_{j}^{-4} f\left(v_{j}^{2}\right)\left(v_{j}^{2}-v_{j-1}^{2}\right) \\
& \leq \sum_{2}^{\infty} \int_{j-1}^{v_{j}^{2}} t^{-2} f(t) d t \\
& =\int_{v_{1}^{2}}^{t^{-2} f(t) d t} \\
& <\infty \text { almost surely, }
\end{aligned}
$$

using the fact that $t^{-1} f(t) \downarrow 0$ and (17).

Finally, $V_{n} \rightarrow \infty$ almost surely,

$$
1-V_{n}^{-2} E\left(x_{n}^{2} \mid F_{n-1}\right)=V_{n}^{-2} V_{n-1}^{2} \leq 1
$$

while

$$
\begin{aligned}
0 \leq V_{n}^{-2} E\left(x_{n}^{2} \mid\right. & \left.F_{n-1}\right)= \\
& =V_{n}^{-2} E\left(x_{n}^{2} I\left(x_{n}^{2} \leq f\left(V_{n}^{2}\right)\right) \mid F_{n-1}\right)+V_{n}^{-2} E\left(x_{n}^{2} I\left(x_{n}^{2}>f\left(V_{n}^{2}\right)\right) \mid F_{n-1}\right) \\
& \leq V_{n}^{-2} f\left(V_{n}^{2}\right)+V_{n}^{-2} E\left(x_{n}^{2} I\left(x_{n}^{2}>f\left(V_{n}^{2}\right)\right) \mid F_{n-1}\right) \\
& \stackrel{\text { a.s. } 0,}{\longrightarrow} 0,
\end{aligned}
$$

since $t^{-1} f(t)+0$ and (11) holds. This verifies condition (13) and 
completes the proof.

\section{References}

[1] C.C. Heyde and D.J. Scott, "Invariance principles for the law of the iterated logarithm for martingales and processes with stationary increments", Ann. Probability 1 (1973), 428-436.

[2] Naresh C. Jain, Kumar Jogdeo and William F. Stout, "Upper and lower functions for martingales and mixing processes", Ann. Probability 3 (1975), 119-145.

[3] Paul Lévy, Processus stochastiques et mouvement brownien (Suivi d'une note de M. Loève. Deuxième édition revue et augmentée. Gauthier-Villars, Paris, 1965).

[4] Jacques Neveu, Mathematical formdations of the calculus of probability (translated by Amiel Feinstein. Holden-Day, San Francisco, California; London; Amsterdam; 1965).

[5] William F. Stout, "A martingale analogue of Kolmogorov's law of the iterated logarithm", $\mathrm{Z}$. Wahrscheinlichkeitstheorie und verw. Gebiete 15 (1970), 279-290.

[6] V. Strassen, "An invariance principle for the law of the iterated logarithm", Z. Wahrscheinlichkeits theorie und verw. Gebiete 3 (1964), 211-226.

[7] Volker Strassen, "Almost sure behavior of sums of independent random variables and martingales", Proc. Fifth Berkeley Sympos. Math. Statistics Probability, Volume II, Part 1, 315-343 (University of California Press, Berkeley and Los Angeles, 1967).

\footnotetext{
Mathematical Institute, Division of Mathematics and Statistics, University of Oxford, CSIRO,

St Giles, Canberra,

Oxford, ACT.

England;
} 